DOI: https://doi.org/10.11144/Javeriana.umed62-3.mnvi

\title{
Manejo de náuseas y vómitos inducidos por quimioterapia en pacientes hematológicos
}

\section{Treatment of Chemotherapy-Induced Nausea and Vomiting in Hematological Patients}

Recibido: 05 Marzo 2021 | Aceptado: 30 Abril 2021

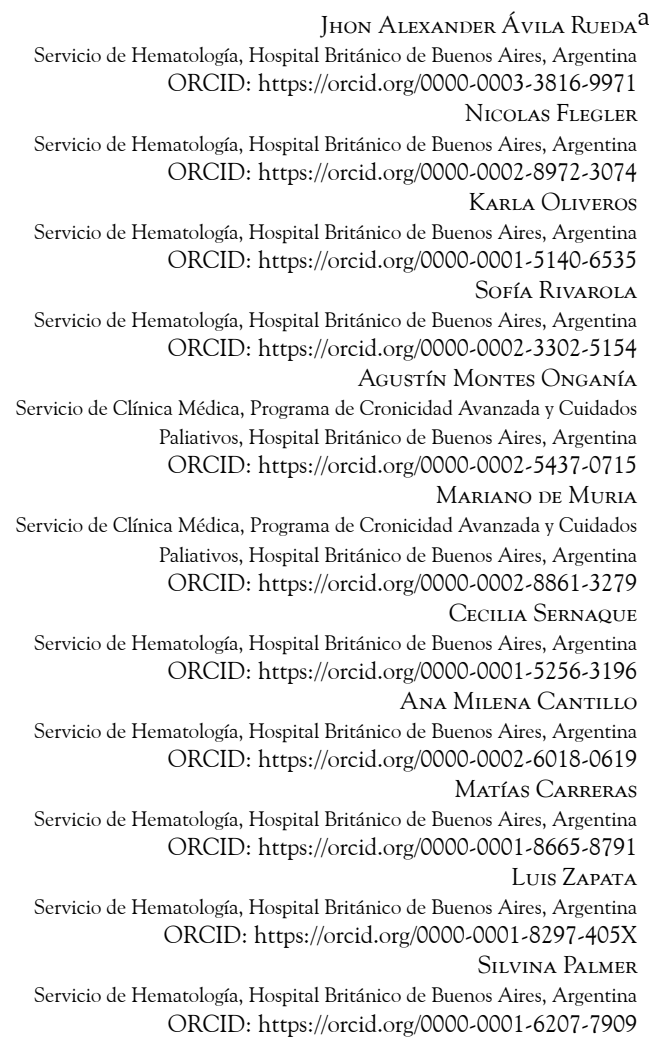

Jhon Alexander Ávila Rueda ${ }^{a}$

Servicio de Hematología, Hospital Británico de Buenos Aires, Argentina ORCID: https://orcid.org/0000-0003-3816-9971

Nicolas Flegler

Servicio de Hematología, Hospital Británico de Buenos Aires, Argentina ORCID: https://orcid.org/0000-0002-8972-3074

Karla Oliveros

Servicio de Hematología, Hospital Británico de Buenos Aires, Argentina ORCID: https://orcid.org/0000-0001-5140-6535

Sofía Rivarola

Servicio de Hematología, Hospital Británico de Buenos Aires, Argentina ORCID: https://orcid.org/0000-0002-3302-5154 Agustín Montes Onganía

Servicio de Clínica Médica, Programa de Cronicidad Avanzada y Cuidados Paliativos, Hospital Británico de Buenos Aires, Argentina ORCID: https://orcid.org/0000-0002-5437-0715

Mariano de Muria

Servicio de Clínica Médica, Programa de Cronicidad Avanzada y Cuidados Paliativos, Hospital Británico de Buenos Aires, Argentina ORCID: https://orcid.org/0000-0002-8861-3279

Cecilia Sernaque

Servicio de Hematología, Hospital Británico de Buenos Aires, Argentina ORCID: https://orcid.org/0000-0001-5256-3196 Ana Milena Cantillo

Servicio de Hematología, Hospital Británico de Buenos Aires, Argentina ORCID: https://orcid.org/0000-0002-6018-0619 Matías Carreras

Servicio de Hematología, Hospital Británico de Buenos Aires, Argentina ORCID: https://orcid.org/0000-0001-8665-8791 LUIS ZAPATA

Servicio de Hematología, Hospital Británico de Buenos Aires, Argentina ORCID: https://orcid.org/0000-0001-8297-405X

Silvina Palmer

Servicio de Hematología, Hospital Británico de Buenos Aires, Argentina ORCID: https://orcid.org/0000-0001-6207-7909

aAutor de correspondencia: jaavilar@gmail.com

Cómo citar: Ávila Rueda JA, Flegler N, Oliveros K, Rivarola S, Montes Onganía A, De Muria M, Sernaque C, Cantillo AM, Carreras M, Zapata L, Palmer S. Manejo de náuseas y vómitos inducidos por quimioterapia en pacientes hematológicos. Univ. Med. 2021;62(3). https://doi.org/10.11144/Javeriana. umed62-3.mnvi

\section{RESUMEN}

Introducción: Las náuseas y los vómitos ( $\mathrm{NyV}$ ) constituyen efectos adversos frecuentemente asociados a la administración de quimioterapia en pacientes hematológicos. Su manejo contribuye a la prevención de riesgos potencialmente asociados, como deshidratación, alteraciones hidroelectrolíticas e insuficiencia renal aguda. Materiales y métodos: Se realizó una revisión de la literatura publicada entre 2014 y 2020 usando las bases de datos PubMed, SciELO, LILACS y Google Académico. Los términos empleados en el motor de búsqueda fueron: náuseas y vómito, quimioterapia y hematología. Los artículos seleccionados en la etapa final requerían la aprobación de al menos $80 \%$ del panel de autores. Resultados: De un total de 100 artículos revisados, se escogieron 30 para elaborar la revisión. En la edición del ensayo se explicaron los mecanismos fisiopatológicos involucrados y, posteriormente, se detallaron los principales esquemas para la prevención y manejo de $\mathrm{NyV}$ inducidos por quimioterapia en función de la evidencia disponible. Conclusión: 
El conocimiento de los mecanismos fisiopatológicos involucrados en el desarrollo de $\mathrm{NyV}$, así como el riesgo de presentación asociado a determinados fármacos citostáticos constituyen pilares para la elaboración de estrategias adecuadas de prevención y manejo.

Palabras clave

náuseas; vómito; quimioterapia; hematología.

\begin{abstract}
Introduction: Nausea and vomiting $(\mathrm{N} \& \mathrm{~V})$ are adverse effects frequently associated with the administration of chemotherapy in hematological patients. The prevention and management of these symptoms contributes to the prevention of potentially associated risks such as: dehydration, electrolyte disturbances and acute renal failure. Materials and methods: A review of the literature published between 2014 and 2020 was carried out using the databases: PubMed, Scielo, LILACS and Google Scholar. The terms used in the search engine were: Nausea and vomiting, chemotherapy and hematology; the articles selected in the final stage required the approval of at least $80 \%$ of the panel of authors. Results: From a total of 100 articles reviewed, 30 were chosen for the preparation of the review. In the edition of the trial, the pathophysiological mechanisms involved were explained and subsequently the main schemes for the prevention and management of chemotherapy-induced $N \& V$ were detailed based on the available evidence. Conclusion: The knowledge of the pathophysiological mechanisms involved in the development of $N \& V$, as well as the risk of presentation associated with certain cytostatic drugs constitute pillars for the development of adequate prevention and management strategies.

Keywords

nausea; vomiting; chemotherapy; hematology.
\end{abstract}

\section{Introducción}

Es ampliamente conocida la aparición de náuseas y vómitos $(\mathrm{NyV})$, secundaria a la administración de fármacos, en particular aquellos pertenecientes a la familia de los citostáticos. Y esto es una de las principales causas que han motivado la suspensión o, en otros casos, el abandono definitivo del tratamiento. Específicamente en el campo de la hematología, el uso extensivo de estos y otros medicamentos, por ejemplo, los anticuerpos monoclonales, se encuentran altamente asociados con la presencia de $\mathrm{NyV}$ (1).

Como consecuencia del mal control de estos síntomas, se pueden desencadenar deshidratación, alteraciones electrolíticas, desnutrición a mediano y largo plazo e insuficiencia renal (2). En vista lo anterior, tanto la prevención como el tratamiento adecuado de los episodios de $\mathrm{NyV}$ constituyen grandes determinantes en el momento de definir la continuidad y el adecuado cumplimiento del régimen terapéutico propuesto a nuestros pacientes.

\section{Materiales y métodos}

Para la elaboración de este artículo se revisó la literatura sobre el tema publicada entre 2014 y 2020. Las preguntas orientadoras durante esta revisión fueron: icuáles son los factores relacionados con la presencia de $\mathrm{NyV}$ en los pacientes oncológicos? ¿Cuáles son los algoritmos vigentes para el tratamiento de $\mathrm{NyV}$ relacionados con quimioterapia? Para ello se consultaron las siguientes bases de datos: PubMed (United States National Library of Medicine), SciELO (Biblioteca Científica Electrónica en Línea), LILACS (Literatura Latinoamericana y del Caribe en Ciencias de la Salud) y Google Académico. Como estrategia de búsqueda, se recurrió al uso de términos clave como: náuseas y vómito, quimioterapia y hematología. Además, se incluyeron búsquedas con los términos antes descritos, traducidos al inglés. Se tomaron en cuenta: metanálisis, guías de práctica clínica y ensayos clínicos aleatorizados en idioma castellano o en inglés. Posteriormente, se usó una segunda estrategia, basada en la revisión manual de cada uno de los artículos preseleccionados, luego de lo cual se escogieron solo aquellos cuyo tema principal fuera: $\mathrm{NyV}$ inducido por quimioterapia en pacientes con enfermedad hematológica. Así mismo, se tomaron como referencia las guías de Sociedad Norteamericana de Oncología Clínica (ASCO) y la Sociedad Europea de Oncología Médica. Luego de seleccionar las referencias bibliográficas, que requirió la aprobación del $80 \%$ del panel de autores, se elaboró el contenido de este artículo, inicialmente bajo la forma narrativa y, posteriormente, analizando las estrategias para el manejo de $\mathrm{NyV}$ según la evidencia disponible. 


\section{Resultados}

Se analizaron un total de 100 artículos, de los cuales se eligieron 40 para una segunda etapa de revisión. Al final se seleccionaron 30 que cumplieron con los criterios de inclusión para formar parte de la revisión. En función de la base de datos empleada, 14 de los artículos escogidos fueron de PubMed; 8, de SciELO; 6, de LILACS, y 2, de Google Académico.

Cada una de las referencias citadas para el manejo de $\mathrm{NyV}$ se clasificaron según el nivel de evidencia planteado por Sackett en los siguientes grados de recomendación: grado A (niveles 1a y 1b): evidencia fuerte (revisión sistemática o metanálisis y ensayos clínicos aleatorizados); grado B ( niveles 2a, 2b, 3a, 3b): evidencia fuerte/moderada (estudios de cohortes y casos y controles con buena calidad metodológica); grado C (nivel 4): evidencia baja (estudios de cohortes y casos y controles con baja calidad metodológica); grado D (nivel 5): evidencia débil (opinión de expertos) (3).

\section{Mecanismo fisiopatológico del desarrollo de náuseas y vómito}

El origen de las $\mathrm{NyV}$ resulta de la interacción entre múltiples estructuras, a saber: el centro reflejo del vómito (CRV), la zona quimiorreceptora gatillo (ZQG) y las aferencias centrales y periféricas de estas dos.

Respecto a la ZQG, se encuentra en el área postrema localizada en el piso del cuarto ventrículo, lugar donde la barrera hematoencefálica no está lo suficientemente desarrollada y la lleva a ser susceptible de recibir estímulos emetogénicos, entre los cuales se incluyen las drogas usadas para el tratamiento con quimioterapia, así como los trastornos metabólicos (por ejemplo, los asociados a elevación de la urea e hipercalcemia). Una vez que se estimula la $Z Q G$, transmite señales de activación al centro reflejo del vómito, que consiste en una amplia red neuronal que integra los estímulos emetogénicos de la ZQC $\mathrm{y}$ otras aferencias provenientes del nervio vago y nervios esplácnicos, glosofaríngeo y aferencias provenientes de la corteza y el sistema vestibular, que se localizan en la formación reticular del tronco encefálico.

En el contexto de las $\mathrm{NyV}$ por quimioterapia, el CRV lo activan receptores serotoninérgicos (5HT), dopaminérgicos $\left(\mathrm{D}_{2}\right)$ y de neurocinina (NK-1), a través de mediadores humorales, donde la estimulación de receptores $5 \mathrm{HT}_{3}, 5 \mathrm{HT}_{2}$ y $\mathrm{D}_{2}$ está asociada al desarrollo de eventos de $\mathrm{NyV}$ agudos y a la estimulación de receptores NK-1, relacionada con episodios tardíos $(4,5)$.

Una vez estimulado el CRV, será el encargado de trasmitir señales eferentes a través de los nervios vago y frénico hacia el centro de salivación, estómago, diafragma y músculos abdominales, con la finalidad de lograr el aumento de la presión dentro de la cámara gástrica y posterior expulsión del contenido intracavitario al exterior $(5,6)$. Si bien en términos generales los mecanismos generadores de NyV se corresponden de forma total o parcial con lo descrito, en la actualidad se tiene poca información acerca de la fisiología del vómito inducido por quimioterapia, aunque se conoce que a través de la estimulación de vías periféricas mediadas principalmente por serotonina y las vías centrales mediadas por neurocinina logran activar el CRV con la consecuente aparición de síntomas (figura 1). 


\section{Figura 1.}

Mecanismos para el desarrollo de náusea y vómito de origen farmacológico
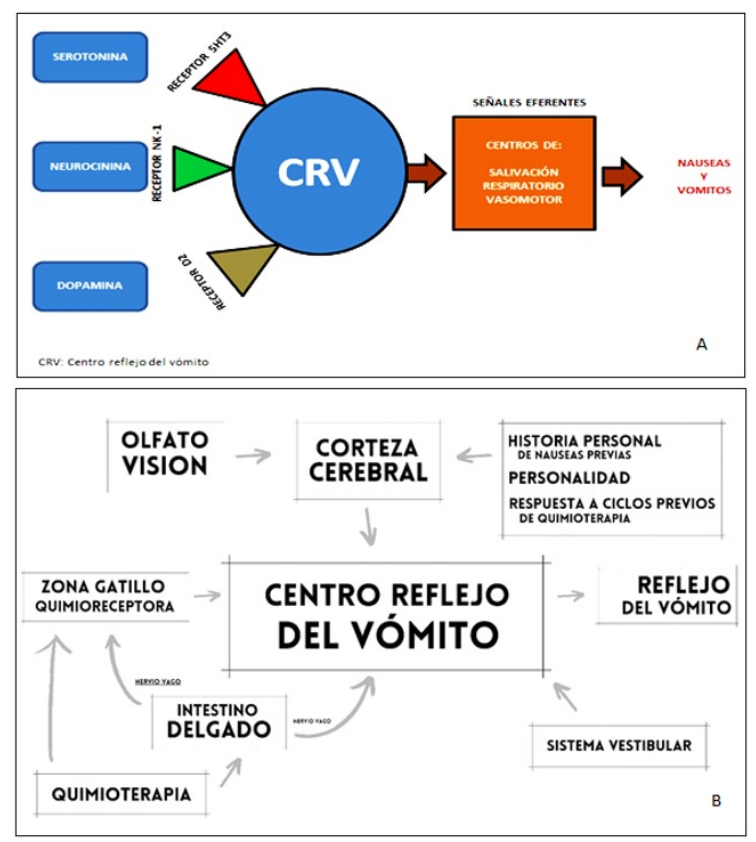

CVR: centro reflejo del vómito.

Clasificación de las náuseas y vómito inducidos por quimioterapia

Agudos: aparecen durante las primeras 24 horas luego de la administración del esquema de quimioterapia (7).

Tardíos: aparecen luego de las 24 horas de administración y, habitualmente, persisten hasta los 5 días posteriores a la infusión (7).

$\mathrm{NyV}$ anticipatorios: obedecen a una respuesta condicionada del individuo luego de haber presentado un episodio de $\mathrm{NyV}$ en ciclos anteriores. Dado que su fisiopatología y tratamiento son distintos, tendrán un apartado específico (8).

$\mathrm{NyV}$ irruptivos: aquellos cuya manifestación se lleva a cabo durante la quimioterapia, a pesar de la instauración de un esquema adecuado de profilaxis antiemética (8).

$\mathrm{NyV}$ refractarios: aquellos cuya aparición persiste en ciclos sucesivos de quimioterapia, a pesar de la administración del esquema de profilaxis antiemética acorde (8).
Factores de riesgo para el desarrollo de las náuseas y los vómitos inducidos por quimioterapia

Existen distintos factores que pueden aumentar la probabilidad de ocurrencia de las $\mathrm{NyV}$ en los pacientes sometidos a tratamiento con quimioterapia. Estos deben evaluarse de forma sistemática para cada paciente en particular y no solamente a la luz del fármaco que se va a utilizar. Con base en lo antedicho, podemos clasificar los factores de riesgo como aquellos dependientes del paciente y aquellos dependientes del esquema de quimioterapia.

\section{Factores de riesgo dependientes del paciente}

Sexo femenino. Es uno de los factores de riesgo con mayor aceptación, dado que ha sido encontrado como un factor de riesgo independiente en múltiples estudios (8-10). El más relevante es el trabajo de Suji et al. (10), en el cual se llevó a cabo una regresión logística multivariada para identificar los factores de riesgo independientes sobre 825 pacientes bajo tratamiento con cisplatino y triple esquema antiemético, incluidos granisetrón o palonosetrón (10).

Edad. Si bien el límite de edad varía entre 55 y 60 años, según los estudios evaluados, existe evidencia de que en pacientes menores de 60 años existe un aumento del riesgo de $\mathrm{NyV}$.

Estatus performance medido por la escala ECOG. El ECOG mayor o igual a 1 se asocia con mayor riesgo de $\mathrm{NyV}$, aunque este hallazgo fue demostrado solo para las náuseas de inicio tardío (11).

Consumo de alcohol. El consumo de alcohol de forma crónica fue encontrado como factor protector para la aparición de $\mathrm{NyV}$ de inicio tardío en hombres. Este hallazgo no presenta explicación por el momento, aunque podría deberse al daño crónico sobre la ZQG inducida por el alcohol.

Historia de cinetosis. Este antecedente fue encontrado en múltiples estudios como un factor de riesgo independiente (11). 
Factores de riesgo relacionados con los medicamentos usados en hematooncología

Existen diferentes subgrupos de medicamentos dentro de la familia de los citostáticos frecuentemente usados en el tratamiento de enfermedades hematológicas. En 2019, la ASCO reunió un grupo de expertos que, a partir de la revisión de la literatura sumada a su experticia clínica, se dieron a la tarea de clasificar los diferentes fármacos empleados en tratamientos oncológicos en función de su potencial emetogénico. Este se dividió en tres categorías (tabla 1): riesgo alto (episodios de $\mathrm{NyV}$ reportados entre el $30 \%$ y el $90 \%$ de los pacientes tratados), riesgo moderado (entre el $10 \%$ y el $30 \%$ ) y riesgo bajo (menos del 10 $\%$ de episodios de $\mathrm{NyV}$ ). Una vez realizado el consenso, se redactó un documento con recomendaciones para el tratamiento de $\mathrm{NyV}$ $(12,13)$.

\section{Tabla 1.}

Clasificación de medicamentos según el riesgo de desarrollar náusea y vómito

\begin{tabular}{|c|c|}
\hline \multicolumn{2}{|c|}{ Riesgo alto } \\
\hline $\begin{array}{l}\text { Alquilantes: } \\
\text { Carmustina } \\
\text { Ciclofosfamida, dosis } \geq 1500 \mathrm{mg} / \mathrm{m}^{2}\end{array}$ & $\begin{array}{l}\text { Antimetabolitos: dacarbacina } \\
\text { Platinos: cisplatino }\end{array}$ \\
\hline \multicolumn{2}{|c|}{ Riesgo moderado } \\
\hline $\begin{array}{l}\text { Alquilantes: } \\
\text { Bendamustina } \\
\text { Busulfán } \\
\text { Ciclofosfamida, dosis }<1500 \mathrm{mg} / \mathrm{m}^{2} \\
\text { Ifosfamida } \\
\text { Thiotepa } \\
\text { Alcaloides de la vinca: vinorelbina } \\
\text { Anticuerpos monoclonales: alemtuzumab } \\
\text { Antimetabolitos: } \\
\text { Citarabina, dosis }>1000 \mathrm{mg} / \mathrm{m}^{2} \\
\text { Clofarabina }\end{array}$ & $\begin{array}{l}\text { Antraciclinas: } \\
\text { Daunorrubicina } \\
\text { Doxorrubicina } \\
\text { Idarrubicina } \\
\text { Compuestos naturales: trióxido de arsénico } \\
\text { Hipometilantes: azacitidina } \\
\text { Inhibidores de tirosina cinasa: imatinib } \\
\text { Platinos: } \\
\text { Carboplatino } \\
\text { Oxaliplatino }\end{array}$ \\
\hline \multicolumn{2}{|c|}{ Riesgo bajo } \\
\hline $\begin{array}{l}\text { Alquilantes: melfalán } \\
\text { Alcaloides de la vinca: vincristina } \\
\text { Anticuerpos monoclonales: } \\
\text { Blinatumumab } \\
\text { Brentuximab } \\
\text { Daratumumab } \\
\text { Elotuzumab } \\
\text { Gemtuzumab ozogamicina } \\
\text { Inotuzumab } \\
\text { Obinutuzumab } \\
\text { Ofatumumab } \\
\text { Pembrolizumab } \\
\text { Rituximab } \\
\text { Antimetabolitos: } \\
\text { Citarabina dosis }<1000 \mathrm{mg} / \mathrm{m}^{2} \\
\text { Cladribrina } \\
\text { Fludarabina } \\
\text { Gemcitabina } \\
\text { Hidroxiurea }\end{array}$ & $\begin{array}{l}\text { Metotrexato } \\
\text { Antraciclinas: doxorrubicina liposomal } \\
\text { Hipometilantes: decitabina } \\
\text { Inhibidores de BCL-2: venetoclax } \\
\text { Inhibidores de histona deacetilasa: } \\
\text { Belinostat } \\
\text { Vorinostat } \\
\text { Inhibidores de proteosoma: } \\
\text { Bortezomib } \\
\text { Carfilzomib } \\
\text { Inhibidores de tirosina cinasa: } \\
\text { Dasatinib } \\
\text { Ibrutinib } \\
\text { Idelalisib } \\
\text { Nilotinib } \\
\text { Inhibidores de topoisomerasa II: } \\
\text { Etopósido } \\
\text { Mitoxantrona } \\
\text { Inmunomoduladores: } \\
\text { Lenalidomida } \\
\text { Talidomida }\end{array}$ \\
\hline
\end{tabular}

Medicamentos empleados con frecuencia para el manejo de náuseas y vómito

$\mathrm{Si}$ bien en la actualidad se tiene conocimiento y disponibilidad de acceso a una variedad de fármacos identificados como antieméticos, la finalidad del presente artículo es mencionar aquellos sobre los cuales se tenga mayor experiencia de uso en pacientes oncohematológicos. Así es como tales medicamentos, en función de su mecanismo de acción, se identifican dentro de los siguientes grupos:

\section{Antagonistas del receptor de serotonina 3}

El mecanismo de acción en este grupo de fármacos se da mediante el bloqueo de receptores tipo 5HT3 localizados a nivel central, además de aquellos ubicados en otras zonas como las fibras vagales aferentes del tracto gastrointestinal $(14,15)$. Pertenecen a este grupo el ondansetrón, el granisetrón, el dolasetrón y el palonosetrón, actualmente disponibles en el país. Su principal efecto es en las náuseas de inicio agudo. Los efectos adversos principales para tener en cuenta de este grupo de drogas son la cefalea, la constipación y la prolongación del intervalo QT corregido (QTc) (16).

Algunas guías de manejo internacional ubican al palonosetrón como el antagonista del receptor 5HT3 de elección, principalmente por su vida media prolongada, por la menor tasa de prolongación del intervalo QTc y por estudios que justifican una mayor tasa de respuesta completa de las náuseas de inicio agudo $(17,18)$. Sin embargo, algunos estudios han demostrado que el ondansetrón no es inferior al palonosetrón, motivo por el que puede utilizarse el primero como primera línea, dado que se encuentra más disponible en nuestro medio(18).

\section{Antagonistas del receptor de neurocinina}

Estos medicamentos inhiben selectivamente los receptores de la sustancia $\mathrm{P}$ (neurocinina $1 \mathrm{o}$ 
NK-1), localizados en el tronco encefálico y relacionados con los eventos de emesis tardía, por lo que su principal virtud reside en su capacidad de bloquear episodios de $\mathrm{NyV}$ que puedan aparecer tras 24 horas o más de administrada la quimioterapia (19). Dentro de este grupo están incluidos: aprepitant, fosaprepitant, netupitant y rolapitant. En nuestro medio, el más utilizado es el aprepitant (20).

El aprepitant, en combinación con dexametasona y granisetrón, demostró ser superior al esquema sin aprepitant para lograr la respuesta completa en el caso de las $\mathrm{NyV}$ tardíos en pacientes que recibieron quimioterapia con alto riesgo de emetogenicidad, aunque no mostró diferencias en las $\mathrm{NyV}$ agudas $(20,21)$. Otro trabajo demostró hallazgos similares para el fosaprepitant en dosis única de $150 \mathrm{mg}$ por vía endovenosa en combinación con ondansetrón y dexametasona para pacientes con quimioterapia con moderada emetogenicidad (22).

Por último, merece la pena mencionar el trabajo realizado por Hesketh et al. (21), en 2014, que evaluó la combinación de netupitant en dosis de 100, 200 o 300 mg más 0,5 mg de palonosetrón frente a 0,5 mg orales de palonosetrón en pacientes que recibieron quimioterapia con alto riesgo de emetogenicidad. El netupitant fue superior al palonosetrón en las tres dosis para prevenir las náuseas tardías, aunque el mayor beneficio se vio en los $300 \mathrm{mg}$. Respecto a la seguridad, los efectos adversos de los antagonistas del receptor de neurocinina 1 han demostrado ser seguros en todos los trabajos antedichos, y los efectos adversos más frecuentes son la fatiga, la constipación y, en algunos casos, diarrea como efecto paradójico (23).

En resumen, los antagonistas del receptor de neurocinina demostraron ser más efectivos que la terapia doble (dexametasona y antagonistas del receptor de serotonina) e igualmente seguros para la profilaxis de las $\mathrm{NyV}$ tardíos en esquemas de moderada y alta emetogenicidad. La respuesta preferencial sobre las $\mathrm{NyV}$ tardíos es consistente con el mecanismo fisiopatológico de los mismos (24).

\section{Dexametasona}

La dexametasona demostró aumentar el efecto de los antagonistas del receptor de serotonina 3 tanto en las $\mathrm{NyV}$ agudas como en las tardías. La inhibición del CYP3A4, producida por aprepitant y netupitant, debe tenerse en cuenta, porque aumenta las concentraciones de la dexametasona (20). La principal limitante de este fármaco se debe a los efectos adversos, principalmente al insomnio que produce en las dosis que suelen utilizarse para el tratamiento de las $\mathrm{NyV}$ (tabla 2).

Tabla 2.

Esquemas para tratamiento de náuseas y vómito según el riesgo de presentación

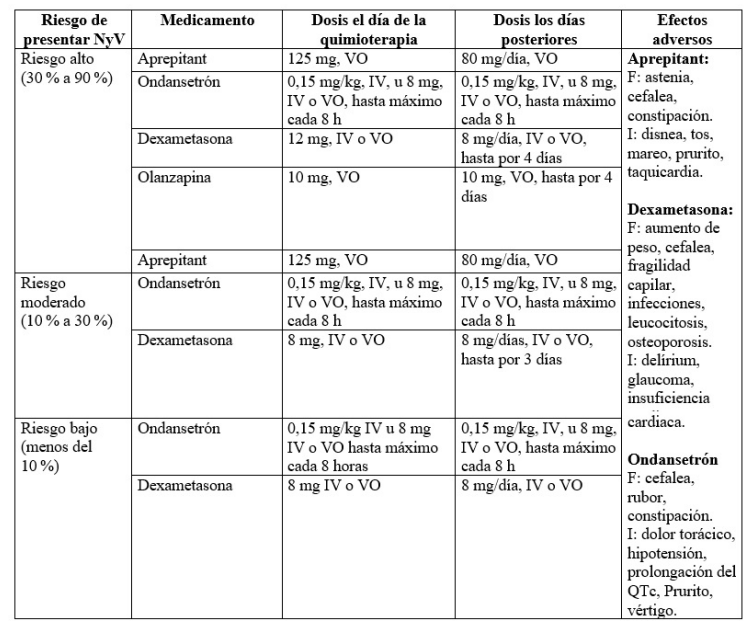

NyV: náuseas y vómitos; IV: intravenoso; VO: vía oral; F: frecuente; I: infrecuente.

Antagonistas de la dopamina

$\mathrm{Su}$ efecto es mediado por el bloqueo de los receptores de dopamina, particularmente los D2 de estimulación quimioceptora, así como los localizados en el CRV. La metoclopramida pertenece a esta familia de medicamentos (25). Es importante mencionar que la metoclopramida no suele recomendarse en la profilaxis, dado que las dosis que han demostrado efecto favorable son de $20 \mathrm{mg}$ cada 6 horas, y estas presentan riesgo de efectos extrapiramidales. 


\section{Otros fármacos}

Dentro de este grupo podemos clasificar a los benzodiacepinas y a los antipsicóticos, como la olanzapina y el haloperidol, cuyo efecto sobre los episodios de $\mathrm{NyV}$ es de leve a moderado, si se usan de forma individual; sin embargo, se ha demostrado que su asociación con regímenes terapéuticos en los que se incluyan antagonistas de 5 HT3 o NK-1 contribuyen a potenciar de modo global el efecto antiemético $(26,27)$.

Esquemas para la prevención y manejo de náuseas y vómito asociado a quimioterapia

Para la edición de este apartado se tomó en cuenta nuevamente la clasificación de medicamentos según su grado de asociación con $\mathrm{NyV}$ emitidas por la ASCO y la revisión de la literatura realizada, y a partir de ello se definió el manejo más apropiado según cada caso. Respecto de los esquemas terapéuticos empleados, se evidenció en toda la literatura consultada que estos guardaban un alto grado de similaridad; por otra parte, se incluyeron solo medicamentos disponibles en el país. Por ello, en función del riesgo de desarrollar $\mathrm{NyV}$ se describen a continuación los siguientes esquemas $(13,28,29)$ :

Riesgo alto:

Esquema de manejo propuesto: uso combinado de cuatro fármacos (nivel 2 grado de recomendación B):

1. Antagonista de receptor de neurocinina $(\mathrm{NK} 1)^{\mathrm{A}}$

2. Antagonista del receptor de serotonina $(5 \mathrm{HT} 3)^{\mathrm{A}}$

3. Dexametasona ${ }^{\mathrm{A}}$

4. Olanzapina ${ }^{\mathrm{A}, \mathrm{B}}$

Riesgo moderado:

Esquema de manejo propuesto: uso combinado de tres fármacos (nivel 2 grado de recomendación B):
1. Antagonista de receptor de neurocinina $(\mathrm{NK} 1)^{\mathrm{A}}$

2. Antagonista del receptor de serotonina $(5 \mathrm{HT} 3)^{\mathrm{A}}$

3. Dexametasona ${ }^{\mathrm{A}}$

Riesgo bajo:

Esquema de manejo propuesto: uso de un fármaco entre dos opciones disponibles (nivel 2 grado de recomendación B):

1. Antagonista del receptor de serotonina $(5 \mathrm{HT} 3)^{\mathrm{A}}$

2. Dexametasona ${ }^{\mathrm{A}}$

La letra A en superíndice indica que se pueden ver las opciones de medicación disponible y dosis en la tabla 2, que se pueden remplazar por lorazepam o alprazolam, según el criterio médico.

En el caso de tratamientos con quimioterapia de dos o más días de duración, es recomendable la continuación del esquema para la prevención de las $\mathrm{NyV}$ hasta dos días luego de finalizado el tratamiento oncológico.

\section{Náuseas y vómitos anticipatorios}

Pueden afectar entre el $8 \%$ y el $14 \%$ de los pacientes según el número de ciclo de quimioterapia evaluado. Los factores de riesgo más relevantes para el desarrollo de este tipo de náuseas son la aparición de $\mathrm{NyV}$ en el ciclo anterior y la presencia de ansiedad (28).

Dado su mecanismo fisiopatológico, suelen ser resistentes al tratamiento con antieméticos, por lo que el mejor método profiláctico es el adecuado manejo de las $\mathrm{NyV}$ durante cada ciclo.

El tratamiento farmacológico con mayor evidencia para este tipo de $\mathrm{NyV}$ consiste en el uso de benzodiacepinas, principalmente con el uso de lorazepam o alprazolam (30).

Náuseas y vómitos refractarios

En estos pacientes es principal determinar aquellos factores de riesgo dependientes de ellos que puedan desempeñar algún rol en la 
refractariedad, a pesar del tratamiento indicado. Dentro de estos factores de riesgo, debe considerarse la posibilidad de un componente de ansiedad en relación con la refractariedad. Si ese es el caso, los pacientes pueden beneficiarse del agregado de lorazepam o alprazolam.

Además de lo antedicho, si el paciente no estaba bajo un régimen de cuatro drogas, debe considerarse el agregado de olanzapina al esquema de profilaxis, dado que se ha demostrado que el agregado de esta última a un régimen de triple terapia puede lograr una eficacia de hasta el $70 \%$ en el contexto de las $\mathrm{NyV}$ refractarias.

En caso de no lograr el control de los síntomas con el agregado de olanzapina o si el paciente ya presentaba un esquema con cuatro drogas, se puede rotar el antagonista de receptores de serotonina y el de neurocinina. Una buena opción es la combinación de 0,5 mg de palonosetrón con $300 \mathrm{mg}$ de netupitant, aunque existe escasa evidencia de que esta rotación presente mayor efectividad (28-30).

\section{Conclusión}

Las $\mathrm{NyV}$ inducidas por quimioterapia representan un síntoma altamente incómodo para los pacientes y, además, se asocian con la posibilidad de complicaciones clínicas producto de la deshidratación o a la aparición de $\mathrm{NyV}$ anticipatorios, que pueden ser de difícil manejo. Desde esta perspectiva, se han desarrollado diferentes guías de manejo evocadas al abordaje de pacientes oncológicos en general; sin embargo, desde el punto de vista de la hematología, el desarrollo durante los últimos 10 años de un número considerable de nuevos fármacos para el tratamiento de enfermedades oncohematológicas hace necesario el desarrollo de trabajos enfocados en el abordaje de $\mathrm{NyV}$ relacionados con fármacos de uso común para esta especialidad. La creación de un algoritmo acorde para la prevención o tratamiento requiere una estrategia basada en la fisiopatología de las náuseas y vómitos. Igualmente, es importante la consideración de planes de tratamiento adaptados al riesgo de desarrollo de $\mathrm{NyV}$ según el agente específico.

\section{Fuentes de financiación}

ninguna

\section{Conflicto de intereses}

Todos los autores firmantes, declaramos no poseer conflictos de interés.

\section{Referencias}

1. Clark-Snow R, Affronti ML, Rittenberg CN. Chemotherapyinduced nausea and vomiting (CINV) and adherence to antiemetic guidelines: results of a survey of oncology nurses. Support Care Cancer. 2018;26(2):557-64. https://doi.org/10. 1007/s00520-017-3866-6

2. Erretiz López G, Cuéllar Guzmán LF, Cárdenas Herrera N. Clasificación y tratamiento de náusea y vómito postoperatorio en el paciente oncológico sometido a cirugía. Rev Chil Anest. 2020;49(2):223-36. https://doi.org/10. 25237/revchilanestv49n02.07

3. Camaño-Puig R. Evidencia científica, metodología y clasificaciones: niveles y recomendaciones. Terapias Complementarias en la esfera pública. Madrid: Dextra; 2019. p. 259-93.

4. Singh P, Yoon SS, Kuo B. Nausea: a review of pathophysiology and therapeutics. Therap Adv Gastroenterol. 2016;9(1):98-112. https ://doi.org/10.1177/1756283X15618131

5. Veiga-Gil L, Pueyo J, LópezOlaondo L. Náuseas y vómitos postoperatorios: fisiopatología, factores de riesgo, profilaxis y tratamiento. Rev Esp Anestesiol Reanim. 2017;64(4):223-32. https://doi.org/10. 1016/j.redar.2016.10.001 
6. Aapro M. CINV: still troubling patients after all these years. Suppor Care Cancer. 2018;26(Suppl 1):5-9. https://doi.org/10.1007/s00520018-4131-3

7. Henson LA, Maddocks M, Evans C, Davidson M, Hicks S, Higginson IJ. Palliative care and the management of common distressing symptoms in advanced cancer: pain, breathlessness, nausea and vomiting, and fatigue. J Clin Oncol. 2020;20;38(9):905-14. htt ps://doi.org/10.1200/JCO.19.00470

8. Miao J, Liu X, Wu C, Kong $\mathrm{H}$, Xie W, Liu K. Effects of acupressure on chemotherapy-induced nausea and vomiting-a systematic review with meta-analyses and trial sequential analysis of randomized controlled trials. Int J Nurs Stud. 2017;70:27-37. https:// doi.org/10.1016/j.ijnurstu.2017.02.014

9. Scotté F, Chastenet M, Leroy P, Aumont L, Goudiaby C, Legeay K, Beuzeboc P. Nausea and vomiting induced by chemotherapy. Rev Prat. 2019;69(1):49-54.

10. Suji D, Suzuki K, Kawasaki Y, Goto K, Matsui R, Seki N. Risk factors associated with chemotherapyinduced nausea and vomiting in the triplet antiemetic regimen including palonosetron or granisetron for cisplatin-based chemotherapy: analysis of a randomized, double-blind controlled trial. Support Care Cancer. 2019(3):1139-47. https://doi.org/10.10 07/s00520-018-4403-y

11. Hayashi $T$, Shimokawa M, Matsuo K, Miyoshi T, Toriyama Y, Yokota C, et al. Risk factors for delayed chemotherapyinduced nausea and vomiting with low-emetic-risk chemotherapy: a prospective, observational, multicenter study. Cancer Manag Res. 2018;10:4249-55. https://doi.org/10.21 47/CMAR.S176574
12. Alamri A, Alawlah YA, Qiao Y, Wang J. A retrospective review of treatment patterns of antiemetic agents for chemotherapy-induced nausea and vomiting. SAGE Open Med. 2018;6:2050312118767234. https://doi .org/10.1177/2050312118767234

13. Hesketh PJ, Kris MG, Basch E, Bohlke K, Barbour SY, Clark-Snow RA, et al. Antiemetics: American Society of Clinical Oncology Clinical Practice Guideline Update. J Clin Oncol. 2017 Oct 1;35(28):3240-61. https://doi.org/ 10.1200/JCO.2017.74.4789

14. Vaid AK, Gupta S, Doval DC, Agarwal S, Nag S, Patil P, et al. Expert Consensus on Effective Management of Chemotherapy-Induced Nausea and Vomiting: An Indian perspective. Front Oncol. 2020 Mar 27;10:400. https://do i.org/10.3389/fonc. 2020.00400

15. Viale PH. Chemotherapy-induced nausea and vomiting: updates and recommendations. J Adv Pract Oncol. 2018 Mar;9(2):150-2.

16. Brygger L, Herrstedt J; Academy of Geriatric Cancer Research (AgeCare). 5-Hydroxytryptamine3 receptor antagonists and cardiac side effects. Expert Opin Drug Saf. 2014 Oct;13(10):1407-22. https://doi.org/10 .1517/14740338.2014.954546

17. Parathoduvil AA, Sisupalan A, Rema PL. Comparison of antiemetic effectiveness of palonosetron versus ondansetron in patients on cancer chemotherapy: a prospective observational study in South Indians. J Clin Diagn Res. 2017(5):10-14. https://doi.org/10.7860 /JCDR/2017/25129.9818

18. Atil V, Prasada H, Prasad K, Shenoy UV. Comparison of antiemetic efficacy and safety of palonosetron vs ondansetron in the prevention of chemotherapy-induced nausea and 
vomiting in children. J Community Support Oncol. 2015(6):209-13.

19. Jordan K, Blättermann L, Hinke A, Müller-Tidow C, Jahn F. Is the addition of a neurokinin-1 receptor antagonist beneficial in moderately emetogenic chemotherapy? -a systematic review and meta-analysis. Support Care Cancer. 2018;26(1):21-32. doi: $10.1007 / \mathrm{s} 00520-017-3857-7$

20. $\mathrm{Hu} \mathrm{Z}$, Cheng $\mathrm{Y}$, Zhang $\mathrm{H}$, Zhou C, Han B, Zhang Y, et al. Aprepitant triple therapy for the prevention of chemotherapy-induced nausea and vomiting following highdose cisplatin in Chinese patients: a randomized, double-blind, placebocontrolled phase III trial. Support Care Cancer. 2014(4):979-87. https://doi.or g/10.1007/s00520-013-2043-9

21. Hesketh PJ, Rossi G, Rizzi G, Palmas M, Alyasova A, Bondarenko I, et al. Efficacy and safety of NEPA, an oral combination of netupitant and palonosetron, for prevention of chemotherapy-induced nausea and vomiting following highly emetogenic chemotherapy: a randomized doseranging pivotal study. Ann Oncol. 2014(7):1340-6. https://doi.org/10.109 3/annonc/mdu110

22. Weinstein $\mathrm{C}$, Jordan $\mathrm{K}$, Green SA, Camacho E, Khanani S, Beckford-Brathwaite E, et al. Single-dose fosaprepitant for the prevention of chemotherapy-induced nausea and vomiting associated with moderately emetogenic chemotherapy: results of a randomized, doubleblind phase III trial. Ann Oncol. 2016;27(1):172-178. https://doi.org/10 $.1093 /$ annonc/mdv482

23. Kamen C, Tejani MA, Chandwani $\mathrm{K}$, Janelsins M, Peoples AR, Roscoe JA, et al. Anticipatory nausea and vomiting due to chemotherapy. Eur J Pharmacol.
2014; 722:172-79. https://doi.org/10.1 016/j.ejphar.2013.09.071

24. Navari RM. Treatment of breakthrough and refractory chemotherapy-induced nausea and vomiting. Biomed Res Int. 2015:595894. https://doi.org/10.1155/ 2015/595894

25. Masiongale AJ, Garvin JT, Murphy MJ, Hooper VD, OdomForren J, Masiongale JI, et al. Reexamining metoclopramide's role in the prevention of postoperative nausea and vomiting: a secondary analysis. AANA J. 2018;86(3):213-9.

26. Rasheed MA, Sarkar A, Arora V. Evaluation of efficacy of metoclopramide, dexamethasone and their combination for the prevention of postoperative nausea and vomiting (PONV) in patients undergoing cesarean section. Anesth Crit Care. 2019;1(1):1-9. https://doi.org/10.2650 2/acc.001

27. Storz E, Gschwend JE, Retz M. Chemotherapy-induced nausea and vomiting: current recommendations for prophylaxis. Urologe A. 2018;57 (5):532-542. https://doi.org/10 $.1007 / \mathrm{s} 00120-018-0606-6$

28. Umar RM. Drug-drug interactions between antiemetics used in cancer patients. J Oncol Sci. 2018;4(3):142-6. https://doi.org/10.10 16/j.jons.2018.07.003

29. Roila F, Molassiotis A, Herrstedt J, Aapro M, Gralla RJ, Bruera E, van der Wetering M. MASCC and ESMO guideline update for the prevention of chemotherapyand radiotherapy-induced nausea and vomiting and of nausea and vomiting in advanced cancer patients. Ann Oncol. 2016;27:v119-v133. https://doi.org/10. 1093/annonc/mdw270 
30. Malik IA, Khan WA, Qazilbash M, Ata E, Butt A, Khan MA. Clinical efficacy of lorazepam in prophylaxis of anticipatory, acute, and delayed nausea and vomiting induced by high doses of cisplatin. A prospective randomized trial. Am J Clin Oncol. 1995(2):170-5. https://doi.org/10.1097 /00000421-199504000-00017 